\title{
PRESENTACIÓN
}

\section{Sobre los números extraordinarios en materia de Derecho del trabajo y de la Seguridad Social}

\author{
Eva María Blázquez Agudo \\ Directora de Femeris. Universidad Carlos III de Madrid \\ orcid id: 0000-0002-8214-1960
}

doi: https://doi.org/10.20318/femeris.2019.4762

La revista Femeris nació con vocación de colaborar en la difusión de los estudios académicos con perspectiva de género en todas las áreas de conocimiento. Dirigidos por tal objetivo, desde 2016 se han venido publicando dos números anuales que han incluido trabajos en la materia des de una visión multidisciplinar.

Como ya se puso en evidencia en la Editorial el número anterior, a partir de 2019 se amplia a tres el número de volúmenes que se van a publicar anualmente en la revista. Dos serán generales con una visión multidisciplinar y se publicarán en el mes de enero y septiembre. El otro número, que verá la luz en mayo, versará sobre el tema de la igualdad en el ámbito concreto del Derecho del Trabajo y de la Seguridad Social.

Recientemente, en el Congreso Anual celebrado en Santiago en mayo de 2018, la Asociación Española de Derecho del Trabajo y de la Seguridad Social (en adelante, AEDTSS) ha nombrado una la Comisión Gestora de Igualdad, que tiene por objeto poner en funcionamiento lo que será la Comisión Gestora de Igualdad, un organismo que nace con la vocación de desarrollar una actuación de defensa de los derechos a la igualdad y a la no discriminación en el ámbito de las relaciones laborales. Como una de sus funciones es la acción divulgativa con el fin de favorecer el debate y el estudio sobre este ámbito, se asumió la necesidad de organizar una publicación, donde incluir estos estudios.

En este marco, se ha formado un Convenio entre la Universidad Carlos III y la AEDTSS que busca el apoyo a la difusión de trabajos en materia de igualdad, cuyo cauce serán los números especiales de Femeris. Como la función de la mencionada Comisión de Igualdad va más allá de la discriminación por razón de género, sino que también incluye en otras tales como discriminación por razón de edad, raza o etnia, discapacidad u orien-

*evamaria.blazquez@uc3m.es 
tación sexual, el campo de la revista también se extenderá a esta pretensión en dichos números especiales.

La Comisión de Igualdad de la AEDTSS conformará el Consejo Editorial del número anual especial que se publique cada mayo, coincidiendo con el Congreso Anual de la Asociación. También se ha conformado una Comité Científico diferente para este número especial que está integrado por prestigiosas especialistas en materia de igualdad en el ámbito académico, judicial y social.

La división de la Revista tiene sus peculiaridades en estos números especiales. Se mantendrán dos secciones (artículos y misceláneas) sometidas a revisión por pares. En la sección de artículos, además, se publicará cada año el Premio 8 de marzo a la mejor investigación sobre Igualad de Mujeres que se concede cada año por la AEDTSS. El premio citado es concedido de acuerdo con la revisión por pares realizada por la Comisión de Igualdad. Por otra parte, bajo la rúbrica de Misceláneas se publicarán los artículos que versen sobre materias distintas a la igualdad de género.

Se añade una nueva sección, que solo se incluirá en los números especiales, que se denominada Crónicas Jurisprudenciales. Esta parte, en general, constará de tres aportaciones en materia de igualdad. La primera una crónica sobre la jurisprudencia europea a cargo de Jean Jacqmain, profesor jubilado de la Universidad Libre de Bruselas; otra sobre jurisprudencia del Tribunal Constitucional a cargo de Ricardo Ron Latas, Profesor Titular de Universidad de Derecho del Trabajo y Seguridad Social de la Universidade da Coruña y Magistrado Suplente de la Sala de lo Social del TSJ Galicia; y, por último, una sobre la jurisprudencia de los Tribunales Superiores de Justicia, a cargo de la sección juvenil de la AEDTSS.

Por último, se mantienen las secciones sobre Referencias bibliográficas en la materia y Varia. Estas últimas no son sometidas a revisión por pares. En la primera se aceptan recesiones sobre trabajos en la materia (libros o artículos) y en la segunda se incluyen otras cuestiones como conclusiones sobre jornadas o estudios multidisciplinares más allá del Derecho del Trabajo y de la Seguridad Social.

Ya centrándonos en este primer número especial, se incluyen como artículos, en primer lugar, "El RDL 6/2019 para la garantía de la igualdad de trato y de oportunidades entre mujeres y hombre en el empleo y la ocupación: Dios y el diablo en la tierra del sol". Bajo este sugerente título, la catedrática de Derecho del Trabajo y de la Seguridad Social de la Universidad de Valencia, María Amparo Ballester Pastor, ilumina el camino del análisis de las novedades en materia de igualdad, realizando un profundo examen de las nuevas instituciones y adelantando los problemas jurídicos que se van a plantear en su puesta en marcha. En segundo lugar, el magistrado del TSJ de Galicia, sala de lo social, Fernando Lousada Arochena examina los vaivenes de la jurisprudencia más reciente en el ámbito de la igualdad de género con su artículo "Encuentros y desencuentros entre el TEDH y el TJUE en materia de igualdad de género".

Del siguiente artículo es autora Carmen Sánchez Triguero, Catedrática de Derecho del Trabajo y de la Seguridad Social de la Universidad de Murcia, cuyo título es "Concepto y fundamentación jurídica de las acciones positivas y la promoción profesional de las mujeres en la jurisprudencia del TJUE", donde con gran destreza nos dirige por el iter de la 
jurisprudencia europea en la materia. Patricia Nieto Rojas, profesora doctora de Derecho del Trabajo y de la Seguridad Social de la Universidad Carlos III, aporta otro sugerente artículo, cuyo título es "La promoción profesional de las mujeres en el mercado de trabajo español: propuestas para reducir la segregación”. Ambos trabajos tienen su base en sendas ponencias que se presentaron en la Jornada anual sobre Igualdad de Mujeres de la AEDTSS, que este año se celebró el 7 de marzo de 2019 en el CES.

Concepción Sanz Sáez, profesora Asociada Doctora Derecho del Trabajo y Seguridad Social en la UCLM de Ciudad Real, es la autora del artículo titulado" Medidas de protección laboral y de seguridad social para víctimas de violencia de género", donde realiza un estudio en profundidad sobre todas los instrumentos jurídico-laborales de los que disponen las víctimas de violencia de género. Por otra parte, "La discriminación por maternidad y conciliación en el ámbito universitario" es el título del artículo de Alejandra Selma Penalva, profesora Titular de Derecho del Trabajo y de la Seguridad Social de la Universidad de Murcia, que refiere el arduo camino de las profesoras en el ámbito académico cuando son madres. Se complementa esta sección con el artículo de Gratiela Moraru, profesora Asociada de Derecho del Trabajo y la Seguridad Social y Doctoranda de la Universidad de Castilla-La Mancha, que bajo el título "Sobre las proyecciones convencionales de la igualdad en la clasificación y promoción profesionales: luces y sombras”, presentó como comunicación a la Jornada anual sobre Igualdad de Mujeres de la AEDTSS, que este año se celebró el 7 de marzo de 2019, siendo galardonada con el Premio 8 de Marzo sobre Igualdad de Mujeres de la AEDTSS en 2019.

En la sección Misceláneas se publicarán en los números especiales sobre Derecho del Trabajo y de la Seguridad Social las investigaciones que propongan temas de igualdad más allá de la discriminación por razón de sexo. Se inaugura esta sección con el artículo titulado "La discriminación en el trabajo en razón de la orientación sexual en el ordenamiento italiano", cuya autora es Loredana Ferluga, profesora del departamento de derecho de la universidad de Messina.

Como ya se adelantó, completan este número las crónicas de jurisprudencia europea y constitucional y las referencias bibliográficas sobre los libros: "Hacia una reformulación de la violencia de género en el entorno laboral"; "Vosotras bellas, vosotras fuertes" y "Visiones multidisciplinares de la igualdad entre mujeres y hombres". Se cierra el número con la sección Varia, donde se incluye una Crónica sobre la Jornada 8 Marzo de Igualdad de las Mujeres de la AEDTSS, que se celebró el 7 de marzo de 2019 en el CES y el artículo Promoción profesional de las mujeres en la Negociación Colectiva de Esmeralda Sanz Berzal, socióloga de la Secretaría de Acción Sindical de CCOO y vocal por representación de este sindicato de la Comisión Consultiva de Convenios Colectivos, que aporta un riguroso análisis estadístico en la materia que fue base de su ponencia en las Jornadas ya citadas sobre Igualdad de las Mujeres de la AEDTSS en 2019.

En definitiva, en estos números especiales en materia de Derecho del Trabajo y de la Seguridad Social, que ahora se presentan, Femeris sigue apostando por contribuir al avance en la igualdad a través de la difusión de trabajo académicos. Así, que esperamos vuestras colaboraciones también en este ámbito. 
Solamente queda agradecer a la AEDTSS y, en especial, a su presidenta Doña María Emilia Casas Baamonde, su apoyo en la puesta en marcha de estos nuevos números en materia de Derecho del Trabajo y de la Seguridad Social desde el mismo momento que le comenté por primera vez la existencia de este proyecto. De otra manera no hubiese podido culminar esta colaboración, que posteriormente hizo suya el resto de la Junta Directiva y la Comisión Gestora de Igualdad. Es un honor para Femeris poder recoger los trabajos de los miembros de la AEDTSS. 